\title{
KRAS mutation effects on the 2-[18F]FDG PET uptake of colorectal adenocarcinoma metastases in the liver
}

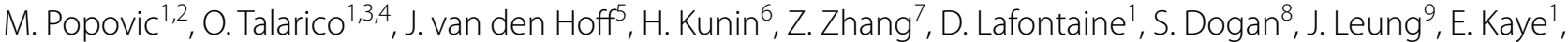 \\ C. Czmielewski ${ }^{1}$, M. E. Mayerhoefer ${ }^{6}$, P. Zanzonico' ${ }^{1}$, R. Yaeger ${ }^{10}$, H. Schöder ${ }^{6}$, J. L. Humm', S. B. Solomon ${ }^{6}$, \\ C. T. Sofocleous ${ }^{6}$ and A. S. Kirov ${ }^{1 *}$ (1)
}

\begin{abstract}
Background: Deriving individual tumor genomic characteristics from patient imaging analysis is desirable. We explore the predictive value of 2-[18F]FDG uptake with regard to the KRAS mutational status of colorectal adenocarcinoma liver metastases (CLM).

Methods: 2-[18F]FDG PET/CT images, surgical pathology and molecular diagnostic reports of 37 patients who underwent PET/CT-guided biopsy of CLM were reviewed under an IRB-approved retrospective research protocol. Sixty CLM in 39 interventional PET scans of the 37 patients were segmented using two different auto-segmentation tools implemented in different commercially available software packages. PET standard uptake values (SUV) were corrected for: (1) partial volume effect (PVE) using cold wall-corrected contrast recovery coefficients derived from phantom spheres with variable diameter and (2) variability of arterial tracer supply and variability of uptake time after injection until start of PET scan derived from the tumor-to-blood standard uptake ratio (SUR) approach. The correlations between the KRAS mutational status and the mean, peak and maximum SUV were investigated using Student's $t$ test, Wilcoxon rank sum test with continuity correction, logistic regression and receiver operation characteristic (ROC) analysis.These correlation analyses were also performed for the ratios of the mean, peak and maximum tumor uptake to the mean blood activity concentration at the time of scan: SUR MEAN $_{\text {, }}$ SUR PEAK and SUR $R_{\text {MAX, }}$ respectively.
\end{abstract}

Results: Fifteen patients harbored KRAS missense mutations (KRAS+), while another 3 harbored KRAS gene amplification. For 31 lesions, the mutational status was derived from the PET/CT-guided biopsy. The Student's $t$ test $p$ values for separating KRAS mutant cases decreased after applying PVE correction to all uptake metrics of each lesion and when applying correction for uptake time variability to the SUR metrics. The observed correlations were strongest when both corrections were applied to $S U R_{\text {MAX }}$ and when the patients harboring gene amplification were grouped with the wild type: $p \leq 0.001 ;$ ROC area under the curve $=0.77$ and 0.75 for the two different segmentations, respectively, with a mean specificity of 0.69 and sensitivity of 0.85 .

Conclusion: The correlations observed after applying the described corrections show potential for assigning probabilities for the KRAS missense mutation status in CLM using 2-[18F]FDG PET images.

Keywords: PET, Colorectal adenocarcinoma, Liver metastases, KRAS mutations

\footnotetext{
*Correspondence: kirova@mskcc.org

${ }^{1}$ Memorial Sloan Kettering Cancer Center, 1275 York Avenue, New York, NY 10065, USA

Full list of author information is available at the end of the article
}

\section{Background}

The value of functional images for personalized therapy is limited by cancer histological and genomic heterogeneity $[1,2]$. Gathering information from medical images 
regarding proliferation rate, differentiation and heterogeneity, or genomic profile of malignant tumors [3, 4] potentially improves selection of the appropriate treatment pathways for targeted therapies while minimizing the need and risks of interventional procedures and maximizing patient comfort [5].

Colorectal cancer $(\mathrm{CRC})$ patients with Kristen rat sarcoma viral gene (KRAS) mutant tumors are associated with lack of response to anti-epidermal growth factor receptor (anti-EGFR) antibody therapy [6-10]. It has been also shown that KRAS mutations are a significant predictor of overall survival in metastatic CRC and of recurrence after surgery or radiofrequency ablation of colorectal cancer liver metastases (CLM) [11, 12]. KRAS mutation has been associated with relatively higher rates of local failure or positive resection margins after thermal ablation or resection of CLM $[11,12]$. Recently, others have found that the coexistence of KRAS mutation with increased 2-[18F]FDG uptake is a negative prognostic factor in primary CRC [13]. KRAS mutation status has a predictive value also for image-guided ablation for lung adenocarcinoma [14].

While primary and metastatic CRC sites have a high concordance for $K R A S$ mutations, meta-analyses suggest the concordance may not be $100 \%$. An initial high (100\%) $K R A S$ genomic concordance has been observed between the primary tumor and secondary lesions in metastatic CRC $[15,16]$. A subsequent meta-analysis showed that the level of concordance may vary, reporting an overall concordance of $94.1 \%$ between 986 pairs of primary and distant metastasis from 17 publications [16]. In a more recent review, Mao et al. [17] have observed a pooled concordance rate of $92 \%$. These data suggest that occasionally KRAS mutant primary tumors harbored KRAS wild-type metastasis and, as such, could potentially benefit from anti-EGFR treatment [17]. Specifically for CLM, these authors found $8.0 \%$ false positive (wild-type or normal expression in primary tumor but mutant or loss of expression in metastases) and 9.7\% false negative (mutant or loss of expression in primary tumor but wild-type or normal expression in metastases) rates [17]. Additionally, patients treated with targeted therapies, including EGFR, HER2 and BRAF targeting combinations, have been reported to develop KRAS mutations at resistance, often in a heterogeneous pattern involving some lesions [18, 19]. Deriving the genomic properties through metabolic imaging of individual lesions may optimize subsequent interventions (Fig. 1).

KRAS mutations appear to be related to the glucose metabolism [20]. Therefore, KRAS mutant lesions may be distinguishable using images of metabolic activity. The manifestation of KRAS mutations in PET images has been studied for non-small cell lung cancer (NSCLC) patients [21, 22] as well as for CRC [13, 23-30]. For NSCLC, one study showed that a multivariate model,

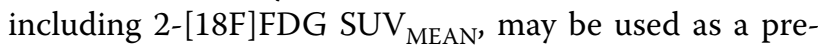
dictive marker for KRAS mutations [21]. However, others found that EGFR mutations correlate with NSCLC PET image features, whereas KRAS mutations do not [22]. Similarly for CRC, one investigation of the effect of KRAS mutations on 2-[18F]FDG uptake of CRC lesions found no correlation [23], while others found statistically significant correlations for primary tumors [13, 24-26]. Several SUV features including $\mathrm{SUV}_{\mathrm{MAX}}$ and $\mathrm{SUV}_{\text {MEAN }}$ were found to significantly correlate with the presence of KRAS mutations for newly diagnosed rectal cancer [27], although predictive value of the 2-[18F]FDG uptake was low. Other investigations showed statistically significant separation of the KRAS-mutated tumors by $\mathrm{SUV}_{\mathrm{MAX}}$ and $S_{U V} V_{\text {PEAK }}$ when a C-reactive protein (CRP) upper threshold of $6 \mathrm{mg} / \mathrm{L}$ was introduced [28], or when $\mathrm{SUV}_{\mathrm{MAX}}$ was used in a decision tree also utilizing CT texture and blood flow, derived from dynamic contrast-enhanced CT [29]. For CRC metastases, SUV MAX was significantly associated with KRAS mutations when considering only tumors larger than $10 \mathrm{~mm}$ in diameter in order to minimize bias due to the partial volume effect (PVE) [30]. Primary tumor SUV $\mathrm{MAX}_{\text {was }}$ found to be significantly higher in CRC subjects with KRAS mutation compared to wild type [13]. None of the above studies were specific to CRC liver metastases (CLM).

We focus on evaluating KRAS effects on 2-[18F]FDG uptake specifically for CLM. In addition, we apply corrections to the 2-[18F]FDG uptake for: (1) partial volume effect and (2) time of tracer uptake from injection to PET scan acquisition. The latter is important for the investigated dataset which contains only PET/CT images obtained in the interventional setting during PET/CTguided ablations or biopsies for which the uptake time can vary. Biopsies and ablations performed under realtime PET/CT guidance are useful to target tumors with poor conspicuity in non-functional/anatomic only imaging [31-35]. If the specimen from a biopsy during such a procedure is subjected to genomic profiling, the molecular and metabolic data are obtained simultaneously for the same lesion, thereby removing uncertainty for the molecular status of the imaged lesion.

\section{Methods \\ Patients}

The records of thirty-seven (37) patients who underwent PET/CT-guided biopsies of colorectal adenocarcinoma liver metastases (CLM) in the period between April 2011 and June 2019 with molecular pathology reports were reviewed under an Institutional Review Board-approved retrospective research protocol. Thirty-nine PET/CT 

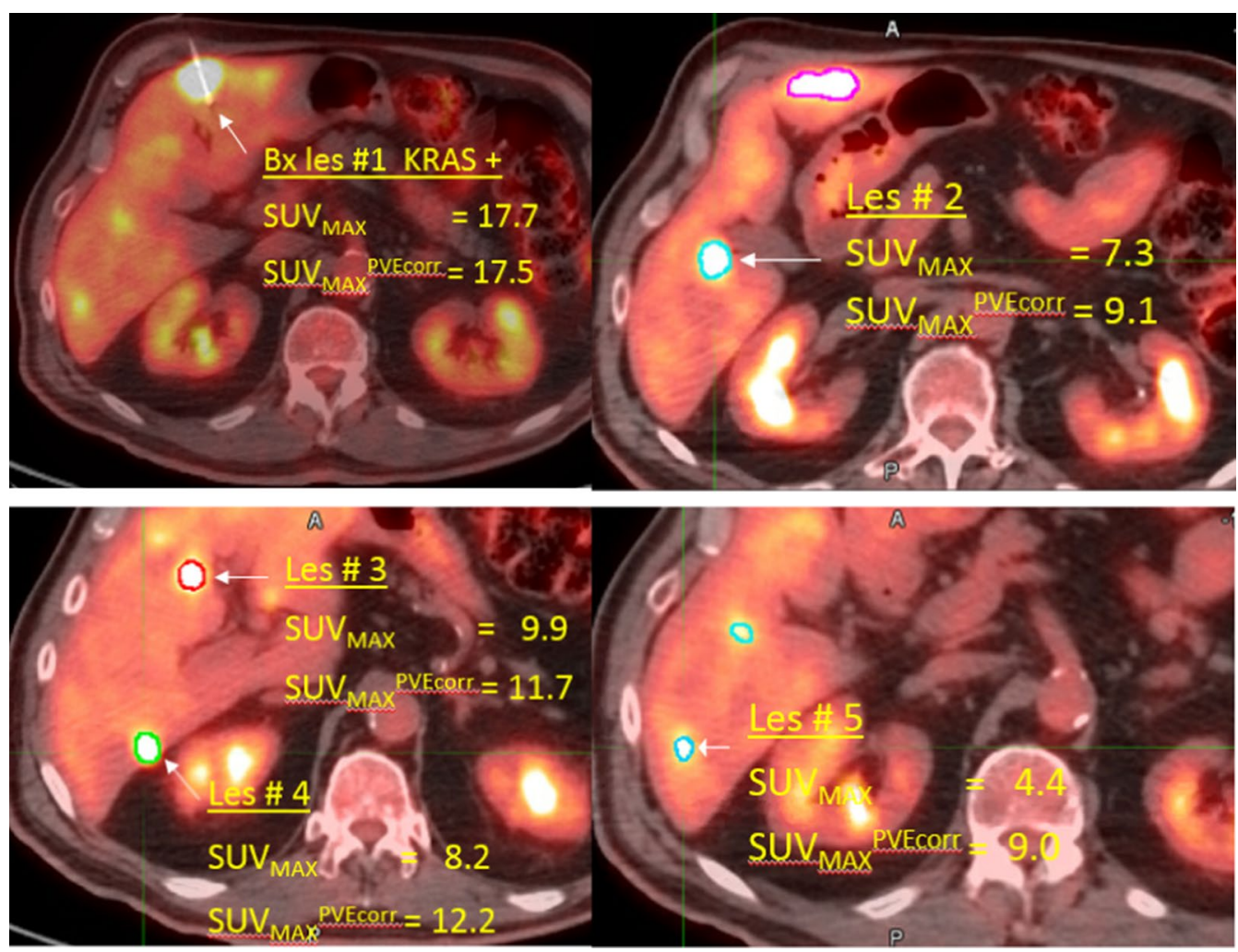

Fig. 1 Fused PET/CT images of multiple liver metastases in a colorectal cancer case. Only one of the lesions was biopsied (top left) and showed KRAS mutant status. Segmentation contours for four lesions are shown. SUV MAX before and after PVE correction is quoted for each lesion. The PVE correction reduces SUV $V_{\text {MAX }}$ for the largest lesion due to the positive bias of maximum uptake values compared to the mean (Fig. 3)

scans were analyzed since two patients underwent a second PET/CT-guided biopsy for new liver lesions, which were also included in the analysis. Altogether 60 CLM were segmented in these 39 interventional PET scans using two different PET segmentation tools. Twelve of the included lesions were in the vicinity of previously treated (ablated or resected) region of the liver.

The KRAS mutational status for each case was extracted from the molecular pathology report of each case. Thirty-one (31) of the 60 lesions had mutational status assigned from the PET/CT-guided procedure. Other lesions in the same cases as well as cases for which the mutational status was known from another specimen were kept in since the probability for mislabeling is small. The investigated dataset is a compromise between reducing the number of potentially mislabeled cases (from about $10 \%$ to about $5 \%$ ) and having a sufficient number of cases for the analysis.

\section{PET/CT-guided biopsies}

In PET/CT-guided biopsy procedures, after the initial $\mathrm{PET} / \mathrm{CT}$ scan, the interventional radiologist places the needle by targeting the PET-avid region of the lesion after which a second CT scan with the needle in place is performed and fused with the pre-insertion PET, $\mathrm{PET}_{\mathrm{pre}}$, for verification of the needle placement [33]. In cases where mis-registration with $\mathrm{PET}_{\text {pre }}$ is suspected due to motion, a second real-time PET with the needle in place may be obtained [36].

All PET/CT-guided biopsies were performed at the same interventional PET/CT scanner (Discovery 690, GE Healthcare, Waukesha, Wisconsin). The average injected 2-[18F]FDG activity (mean \pm SD) was $152.0 \pm 52.4 \mathrm{MBq}$ $(4.1 \pm 1.4 \mathrm{mCi})$. The post-injection residual activity in the syringe was measured only for one protocol case (6.2 MBq for case \#2), while a residual activity of 11.1 $\mathrm{MBq}(0.3 \mathrm{mCi})$ was assumed for all other cases based on our current clinical procedures. Uptake times varied from 40.7 to $205.7 \mathrm{~min}$ (mean $83.8 \mathrm{~min}$, std $34 \mathrm{~min}$ ) due to interventional work specifics: Optimizing the flow of the interventions and patient safety and emergency procedures have a higher priority.

PET scans were centered in the liver and obtained for one or two bed positions consisting of 47 transverse slices, $3.27 \mathrm{~mm}$ thick with 11-slice overlap. Transaxial field of view of $70 \mathrm{~cm}$ and $128 \times 128$ image matrix 
$(5.47 \times 5.47 \mathrm{~mm}$ pixels $)$ were used for all cases. The acquisition time typically varied from 3 to $5 \mathrm{~min}$ per bed for the pre-needle insertion PET scans included in this study. PET scans with breath hold [33] were excluded since their number was very small and breath hold affects the SUV. The reconstruction settings were the same for all scans (time-of-flight on, two iterations, 16 subsets, sharpIR system modeling, post-filter: $6.4 \mathrm{~mm}$, axial filter: heavy).

\section{Molecular pathology}

Molecular pathology reports were obtained from the patients' medical record and reviewed for all 37 patients. If the patients had more than one pathology report, the report from the PET/CT-guided biopsy of the targeted CLM or, if not available, the report closest in time to that biopsy was used. KRAS mutation status was determined by clinically validated molecular assays including the mass spectrometry-based Sequenom technologies [37] test only $(n=5)$ and the next-generation targeted exome sequencing by Memorial Sloan Kettering-Integrated Mutation Profiling of Actionable Cancer Targets (MSKIMPACT) [38] with or without Sequenom $(n=32)$. In addition to KRAS missense mutations, MSK-IMPACT also detects $K R A S$ copy number alterations such as whole gene amplifications. The KRAS mutation status for each case was noted but was not provided to the operators performing tumor segmentation.

\section{PET image analysis}

Sixty CLM were segmented using the PET-edge tool of MIM (MIM Software, Inc., Cleveland, Ohio) and a fixed SUV threshold in Hermes Gold LX (Hermes Medical Solutions, Stockholm, Sweden). Two different researchers performed the segmentations using the two packages and were blinded to the patient mutational status and to each others' results. All automatically generated contours were visually inspected, and small manual corrections were applied if needed. In Hermes, a threshold of SUV $=4.0$ or higher was used: The segmentation thresholds for three higher background cases were increased to avoid unrealistically large volumes.

Mean and peak standard uptake values (SUV, normalized to patient weight) as obtained from the two segmentation methods and the maximum SUVs obtained in Hermes were recorded for all target CLM. When $\mathrm{SUV}_{\text {PEAK }}$ was not available due to a small CLM volume (37 cases out of the 60 lesions in MIM), SUV $\mathrm{SUEAN}_{\text {Mas }}$ used as an approximation.

The tumor-to-blood SUV ratio approach developed by van den Hoff et al. [39] that corrects for variable uptake time from the FDG injection to the PET scan acquisition, variable tracer supply between subjects and technical uncertainty factors [40] was implemented. To determine the 2-[18F]FDG uptake in the blood, we manually segmented the part of the descending aorta visible in the PET images using Hermes. The contours were drawn at least $5 \mathrm{~mm}$ away from the edge of the aorta to minimize PVE effect on SUV mean (Fig. 2).

\section{Partial volume effect correction}

The limited PET resolution causes a partial volume effect which results in loss of accuracy in recovering the true activity especially in small objects. Since at PET resolution most small liver tumors can be approximated by equal volume spheres, an approximate partial volume effect correction can be applied by using the percent contrast $(\mathrm{Q})$ for different-diameter higher activity spheres placed in uniform background as measured according to the NEMA 2.0 protocol for PET scanner acceptance [41]. During PET acceptance, the measurement is performed at a sphere-to-background activity concentration ratio $\mathrm{SBR}=4: 1$. For our purpose, we filled all spheres of the same phantom with $\mathrm{SBR}=2.19: 1(9.284$ and $4.240 \mathrm{kBq} /$

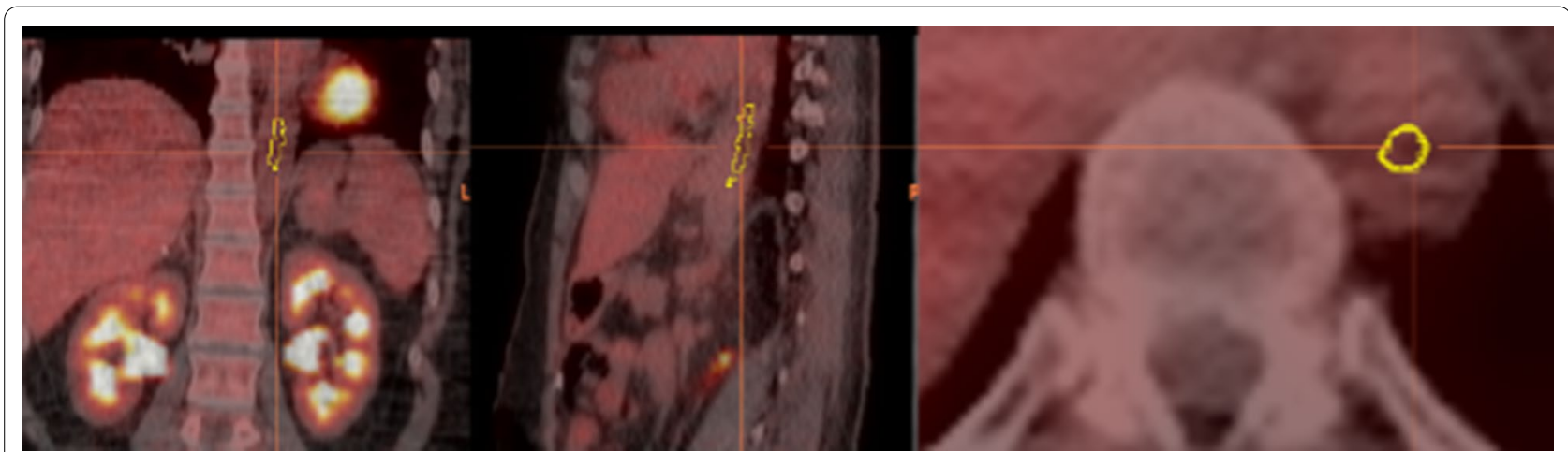

Fig. 2 Cropped fused 2-[18F]FDG PET-CT coronal (left), sagittal (middle) and axial (right) images of the descending aorta with contours used for determining the mean blood SUV (case \# 15: VOI $2.5 \mathrm{~mL}, \mathrm{SUV}_{\text {BLOOD, MEAN }}=1.9$ ) 
$\mathrm{mL}$, respectively) to approximate the mean tumor-tonormal liver ratio in the 39 patient scans. The lung insert in the center of the phantom was also in place with no activity. The phantom was scanned on the same PET scanner where all patients were scanned with two bed positions for $15 \mathrm{~min}$ each to reduce image noise. Spherical VOIs with diameters matching those of the inner diameter of the phantom spheres $(10,13,17,22,28$ and $37 \mathrm{~mm}$ ) were centered in the CT images of the phantom spheres and then copied to the same location in the registered PET images using the Hermes software. Then, the mean, peak and maximum SUV for each sphere were recorded to calculate the respective recovery coefficients as described below.

The equation for contrast recovery in NEMA 2-2018 [41] is

$$
\mathrm{RC}_{\mathrm{MEAN}}^{H, j}=\frac{\frac{C_{\mathrm{MEAN}}^{H, j}}{C_{B, j}}-1}{\frac{a_{H, j}}{a_{B, j}}-1}
$$

where $C_{\mathrm{MEAN}}^{H, j}$ and $c_{B, j}$ are the average counts and $a_{H, j}$ and $a_{B, j}$ are the activity concentrations in hot sphere $j$ and in the background, respectively, which were rewritten in terms of SUV by introducing the respective constants. Then, acknowledging that for the mean background SUV the recovery coefficient is $\mathrm{RC}=1.0$, for the partial volume corrected mean SUV for sphere $j, \mathrm{SUV}_{\mathrm{MEAN}, j}^{\mathrm{PVEC},}$, we obtain:

$$
\mathrm{SUV}_{\text {MEAN }}^{\mathrm{PVEC}, j}=\frac{\mathrm{SUV}_{\text {MEAN }}^{H, j}}{\mathrm{RC}_{\text {MEAN }}^{H, j}}-\mathrm{SUV}_{\text {MEAN }}^{B, j}\left(1 / \mathrm{RC}_{\text {MEAN }}^{H, j}-1\right),
$$

where $\mathrm{SUV}_{\mathrm{MEAN}}^{H, j}$ and $\mathrm{SUV} \mathrm{MEAN}_{\text {ME }}^{B, j}$ are the measured mean SUVs for hot sphere $j$ and the background around it.

In analogy to (1), recovery coefficients can be defined also for the maximum and for the peak measured activities:

$$
\mathrm{RC}_{\mathrm{MAX} \text { or PEAK }}^{H, j}=\frac{\frac{C_{\mathrm{MAX} \text { or PEAK }}^{H, j}-1}{C_{B, j}}}{\frac{a_{H, j}}{a_{B, j}}-1},
$$

which will allow to apply partial volume correction to $S_{U V} V_{\text {PEAK }}$ and $S_{U V} V_{\text {MAX }}$ using:

$$
\begin{aligned}
\mathrm{SUV}_{\text {MAX or PEAK }}^{\text {PVEC } j}= & \frac{\mathrm{SUV}_{\mathrm{MAX} \text { or PEAK }}^{H, j}}{\mathrm{RC}_{\text {MAX or PEAK }}^{H, j}} \\
& -\operatorname{SUV}_{\text {MEAN }}^{B, j}\left(1 / \mathrm{RC}_{\text {MAX or PEAK }}^{H, j}-1\right)
\end{aligned}
$$

The measured recovery coefficients for SUV $\mathrm{SEAN}_{\text {, }}$

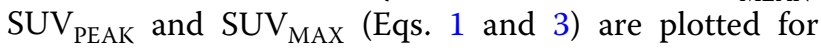
each sphere in Fig. 3. Corrections to these recovery

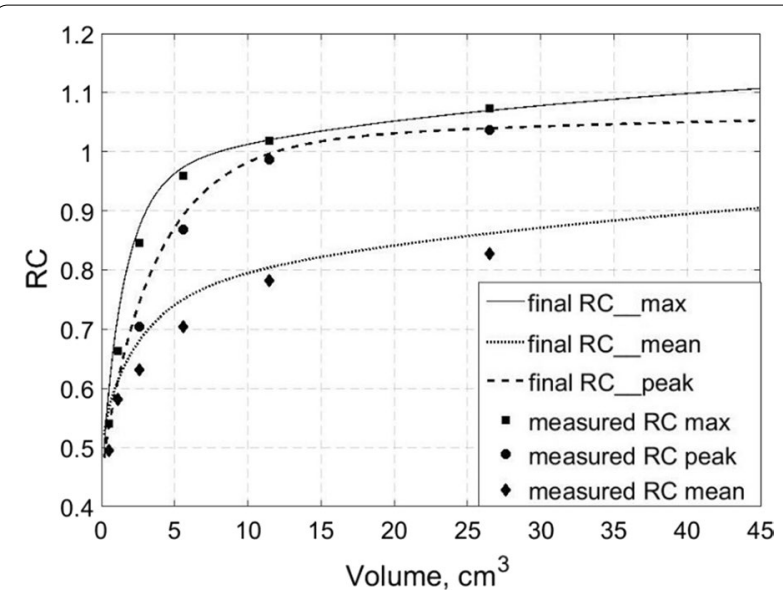

Fig. 3 Recovery coefficients (RC) used for applying PVE correction to the tracer uptake of each lesion. The symbols represent the measured RC for each NEMA NU-2 image quality phantom sphere. The final recovery coefficients used for PVE correction (lines) are obtained by applying a correction for the cold walls of the phantom spheres obtained by using a RC model [42] to compute the recovery coefficient ratio for spheres with and without walls

coefficients were applied for the $1 \mathrm{~mm}$ thickness of the cold sphere walls using a RC model obtained by convolution of the resolution PSF with the spheres given the known sphere-to-background contrast (lines in Fig. 3) [42]. The cold wall correction was obtained from the ratio of the simulated recovery coefficients for the max, peak and mean SUVs for spheres with the same inner diameters with and without $1 \mathrm{~mm}$ walls, respectively. The sphere external diameters were confirmed by caliper measurements, and the wall thickness was verified using a micro-CT scan for some of the spheres. In the simulations, we performed convolution of a symmetrical Gaussian point-spread function with each sphere and used a sphere-to-background ratio of 2.26 , which is close to the midpoint between the ratios in the NEMA phantom and the patients. The cold wall corrections to the $\mathrm{RC}$ ranged up to $11.1 \%$ and $12.8 \%$ for $1 \mathrm{~mL}$ lesions for $\mathrm{SUV}_{\text {MEAN }}$ and $\mathrm{SUV}_{\mathrm{MAX}}$ and were less than $5 \%$ and $0.2 \%$ for volumes larger than $10 \mathrm{~mL}$, respectively. For extending the PVE corrections to the few lesions with volumes larger than the 37 -mm-diameter sphere $(26.52 \mathrm{~mL})$, we followed the RC trends provided by the convolution-based model described above [42]. Note that according to our definition (Eq. 3), the RC for $S U V_{M A X}$ and $S U V_{\text {PEAK }}$ are larger than 1.0 for large lesions due to statistical effects (positive bias of maximum relative to mean).

The approximate $\mathrm{SUV}_{\mathrm{MEAN}}, \mathrm{SUV}_{\text {PEAK }}$ and $\mathrm{SUV}_{\mathrm{MAX}}$ PVE correction for each lesion with volume $V_{\text {les }}, R C_{\text {eff }}$ $\left(V_{\text {les }}\right)$ was obtained by interpolation of the final RC curves obtained after cold wall correction (lines in Fig. 3). The 
mean background SUV for each lesion, $\mathrm{SUV}_{B \text {,lesion, was }}$ measured in two PET slices using doughnut-shaped ROIs manually drawn to avoid the visible spill out from the respective lesion. The PVE-corrected SUV $\mathrm{SEAN}_{\text {, }}$ $S U V_{\text {PEAK }}$ and SUV $V_{\text {MAX }}$ for each lesion are then obtained using $\mathrm{SUV}_{B \text {,lesion }}$ and Eqs. (2) and (4). For lesions close to the periphery of the liver, $S U V_{B, l e s i o n}$ was less than

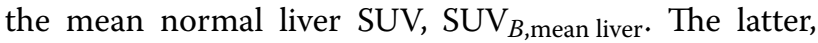

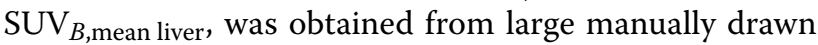
ROIs in normal liver far from the lesions and was used for calculating the tumor-to-liver ratio, $\mathrm{SUVTLR}=\mathrm{SUV}_{\mathrm{PVEC}}$, lesion $/ \mathrm{SUV}_{B \text {, mean liver, }}$ for each lesion.

\section{Uptake time correction}

We employed the uptake time correction method developed by van den Hoff et al. [39] for CRC liver metastases and validated also for other lesions. For calculating the tumor-to-blood standard uptake ratio (SUR) employed by this method, we derived the blood SUV from the 2-[18F]FDG activity in the descending aorta. Since for liver interventions the PET scan is limited to two bed positions (83 slices) providing an axial field of view (FOV) from the inferior to the superior border of the liver, and we extracted $S_{U} V_{\text {blood mean }}$ from an aorta volume at least $0.5 \mathrm{~cm}$ away from the aorta surface to minimize PVE, the aorta ROI volumes were smaller (average analyzed volume $1.8 \mathrm{~mL}$ ) than those previously used [39]. The average SUV values for the descending aorta were $2.2+-0.6 \mathrm{~mL} / \mathrm{g}$. The injection and scan times were automatically extracted from the image headers using an in-house developed tool (DBbrowser). SURs were calculated as $\mathrm{SUR}_{\mathrm{MEAN}}=\mathrm{SUV}$ lesion mean $/ S U V_{\text {blood mean }}, \quad \mathrm{SUR}_{\mathrm{PEAK}}=\mathrm{SUV}_{\text {lesion peak }} /$ $\mathrm{SUV}_{\text {blood mean }}$ and $\mathrm{SUR}_{\mathrm{MAX}}=\mathrm{SUV}_{\text {lesion max }} / \mathrm{SUV} \mathrm{V}_{\text {blood mean }}$, using both MIM and Hermes segmentation contours of the lesions and Hermes contours for $\mathrm{SUV}_{\text {blood mean }}$ A correction for the difference between actual uptake time post-injection, $T$, and standard uptake time $T_{0}=60 \mathrm{~min}$ was applied to each lesion's $\mathrm{SUR}_{\mathrm{MEAN}}$, SUR $_{\text {PEAK }}$ and $S U R_{M A X}$ using the approximations to equations (8) and (10) proposed by van der Hoff et al. [39]: $\quad \mathrm{SUR}_{0}=\frac{T_{0}}{T}\left(\mathrm{SUR}_{T}-V_{r}\right)+V_{r} \approx \mathrm{SUR}_{T} \frac{T_{0}}{T} \quad$ and $\operatorname{SUV}_{0}=\operatorname{SUV}_{T} \frac{\operatorname{SUR}_{0}}{\operatorname{SUR}_{T}}\left(\frac{T_{0}}{T}\right)^{-b} \approx \operatorname{SUV}_{T}\left(\frac{T_{0}}{T}\right)^{1-b} \quad$ with apparent volume of distribution, $V_{\mathrm{r}}=0.53$, and parameter $b=0.313$ as determined previously [39]. By reducing variance, this approach has led to improved correlations in several studies with similar and larger patient cohorts for different cancer types [43-47]. In one of these studies, which used 90 cases with dual time point 2-[18F]FDG PET, the uptake time varied from 56.4 to $197 \mathrm{~min}$ [44].
The lesion volume and the SUV mean, peak and max metrics were extracted immediately after segmentation. Then PVE correction was applied to these values according to the lesion volume. SUVTLR and SUR were calculated using the PVE-corrected mean, peak and max SUV, which was then followed by applying the uptake time correction prior to investigating the correlations.

\section{Results}

\section{Patient demographics and KRAS mutations}

The patient demographics are given in Table 1. KRAS mutations were observed in $15(37.8 \%)$ and copy number variations in $3(8.1 \%)$ of the 37 patients, respectively (Table 2). In 16 of the 18 patients with KRAS mutant tumors (except \#9 and \#23 in Table 2) and 15 of the 19 patients with KRAS wild-type tumors, sequencing was performed on the target CLM from the same PET/CTguided biopsy. Of the 15 missense mutations, eight are in codon G12, four are in codon G13D, two in codon $\mathrm{Q} 61 \mathrm{H}$ and one in codon A146T.

In addition to patient \#11(Table 2), two more patients have developed a KRAS mutation in codon $\mathrm{Q} 61 \mathrm{H}$ after initial testing. Case \#1 had no KRAS mutation in the primary and later, at the time of CLM targeting PET/ CT-guided biopsy, but showed a $\mathrm{Q} 61 \mathrm{H}$ exon 3 KRAS mutation in a different lesion in a remote section of the liver 2.5 years later during preparation for radioembolization. Similarly, case \#31 had no KRAS mutation in an older sigmoid rectum specimen (primary) but exhibited also a codon $\mathrm{Q} 61 \mathrm{H}$ exon 3 KRAS mutation in the targeted CLM at time of the PET/CT-guided biopsy 5 years later. Altogether, both primary tumor and a metastasis were genotyped in 15 cases and the KRAS status was concordant in 13/15 (87\%) cases.

\section{PET image analysis}

The average tumor volumes obtained with the PET-edge algorithm in MIM and with the SUV threshold algorithm in Hermes are $5.3 \mathrm{~mL}$ (min. 0.3, max. 31.3, std. $6.9 \mathrm{~mL}$ )

Table 1 Patient demographics

\begin{tabular}{ll}
\hline Number of patients & $\mathbf{3 7}$ \\
\hline Number of PET/CT images & 39 \\
Age (years) & $56.3 \pm 11.9$ \\
Gender & \\
Male & $27(73 \%)$ \\
Female & $10(27 \%)$ \\
Weight (kg) & $90.3 \pm 17.1$ \\
Ablation with biopsy & 27 \\
Biopsy only & 12 \\
\hline
\end{tabular}


Table 2 KRAS mutations and copy number variations observed in 18 of the 37 patients

\begin{tabular}{|c|c|}
\hline Case \# & KRAS gene alteration and location \\
\hline 2 & G13D (c.38G > A) exon 2 \\
\hline 5 & G12D (c.35G > A) exon 2 \\
\hline 6 & $\mathrm{~A} 146 \mathrm{~T}(\mathrm{c} .436 \mathrm{G}>\mathrm{A})$ exon 4 \\
\hline 7 & $\mathrm{G} 12 \mathrm{~A}(\mathrm{c} .35 \mathrm{G}>\mathrm{C})$ exon 2 \\
\hline 8 & G12D (c.35G > A) exon 2 \\
\hline 9 & Whole gene amplification 12p12.1, FC:2.1 \\
\hline 11 & Q61H (c. 183A > C) exon 3 \\
\hline 14 & G13D (c.38G > A) exon 2 \\
\hline 15 & G12V (c.35G > T) exon 2 \\
\hline 16 & $\mathrm{G} 12 \mathrm{D}(\mathrm{c} .35 \mathrm{G}>\mathrm{A})$ exon 2 \\
\hline 23 & G12D (c.35G > A) exon 2 \\
\hline 24 & G13D (c.38G > A) exon 2 \\
\hline 25 & G12D (c.35G > A) exon 2 \\
\hline 28 & Whole gene amplification 12p12.1, FC: 23.0 (Inv. Panel) \\
\hline 31 & $\mathrm{Q} 61 \mathrm{H}(\mathrm{c} .183 \mathrm{~A}>\mathrm{T})$ exon 3 \\
\hline 32 & G13D (c.38G > A) exon 2 \\
\hline 35 & $\mathrm{G} 12 \mathrm{C}(\mathrm{c} .34 \mathrm{G}>\mathrm{T})$ exon 2 \\
\hline 37 & Whole gene amplification 12p12.1, FC: 8.5 \\
\hline
\end{tabular}

FC, fold change; Inv. Panel, investigational panel

and $6.1 \mathrm{~mL}$ (min. 0.2, max. 43.0, std. $8.5 \mathrm{~mL}$ ), respectively. The average lesion diameter from the two segmentations is $22 \mathrm{~mm}$. Before PVE correction, the mean lesion SUV of all 60 lesions was $6.2 \pm 2.2$ (MIM) and $6.0 \pm 1.6$ (Hermes) and the mean normal liver SUV is $2.7 \pm 0.6$ resulting in mean tumor-to-liver ratio of 2.2 and 2.3 for Hermes and MIM lesion contours, respectively. The mean background liver SUV measured in the vicinity of

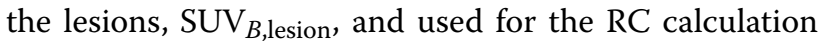
[Eqs. (2) and (4)] is $2.4 \pm 0.7$. The average SUV values obtained for the descending aorta are $2.2 \pm 0.6$. The values for the different uptake metrics corresponding to the two segmentation methods for each mutation status are given in Table 3.

\section{Correlations}

Since only KRAS missense mutations were reliably shown to affect the outcome of anti-EGFR therapy, below we present the results when KRAS gene amplification cases are grouped with wild type. The results when the three cases carrying KRAS gene amplification and no KRAS missense mutations are grouped with KRAS missense mutations are presented in the supplement.

The correlations observed between the investigated SUV metrics and the KRAS mutational status are presented in Table 4 using Student's $t$ test and Wilcoxon rank sum test with continuity correction $p$ values and area under the curve (AUC) of the receiver operating characteristic (ROC). The metrics presented in Table 4 are ordered with decreasing $p$ values from top to bottom. This correlates with the increase in the AUC values which are presented only for the bottom row and the rightmost column.

The mean coefficient of variation of the AUC between the two segmentation methods for $S_{U} R_{M E A N}$, SUR $R_{\text {PEAK }}$ and SUR $\mathrm{MAX}$ with both PVE and uptake time corrections is $4.3,1.5$ and $1.2 \%$ (from the AUC values for SUR $_{\text {PVE+time corr }}$ in Table 4), respectively. ROC curves

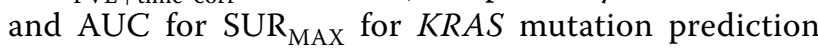

Table 3 Average 2-[18F]FDG SUV metrics ( 1 STD) for KRAS wild-type and missense mutated CLM obtained with two independent volume segmentations (MIM and HERMES)

\begin{tabular}{|c|c|c|c|c|c|c|}
\hline & \multicolumn{2}{|l|}{ Mean uptake } & \multicolumn{2}{|l|}{ Peak uptake } & \multicolumn{2}{|l|}{ Max uptake } \\
\hline & KRAS wild-type & KRAS+ & KRAS wild-type & KRAS+ & KRAS wild-type & KRAS+ \\
\hline SUV (MIM) & $5.70 \pm 1.82$ & $6.91 \pm 2.46$ & $6.56 \pm 2.61$ & $8.29 \pm 4.04$ & $8.63 \pm 3.74$ & $11.79 \pm 5.17$ \\
\hline SUV (HERMES) & $5.63 \pm 1.46$ & $6.65 \pm 1.68$ & $7.09 \pm 2.97$ & $9.21 \pm 4.33$ & $8.63 \pm 3.74$ & $11.79 \pm 5.17$ \\
\hline SUV MIM + PVEC & $7.13 \pm 2.62$ & $8.94 \pm 3.26$ & $7.63 \pm 2.92$ & $9.79 \pm 3.93$ & $9.44 \pm 3.89$ & $13.09 \pm 4.96$ \\
\hline SUV (HERMES) + PVEC & $7.00 \pm 2.12$ & $8.37 \pm 1.86$ & $8.18 \pm 3.17$ & $10.53 \pm 3.95$ & $9.33 \pm 3.66$ & $12.63 \pm 4.68$ \\
\hline SUV (MIM) + PVEC + Time corr & $5.98 \pm 2.39$ & $7.74 \pm 3.18$ & $6.42 \pm 2.74$ & $8.44 \pm 3.66$ & $7.92 \pm 3.57$ & $11.19 \pm 4.46$ \\
\hline SUV (HERMES) + PVEC + Time corr & $5.87 \pm 2.14$ & $7.22 \pm 1.92$ & $6.88 \pm 3.04$ & $8.99 \pm 3.44$ & $7.83 \pm 3.45$ & $10.77 \pm 4.05$ \\
\hline SUVTLR (MIM) + PVEC & $2.64 \pm 1.11$ & $3.54 \pm 1.53$ & $2.81 \pm 1.20$ & $3.92 \pm 1.91$ & $3.51 \pm 1.66$ & $5.28 \pm 2.61$ \\
\hline SUVTLR (HERMES) + PVEC & $2.62 \pm 1.08$ & $3.35 \pm 1.16$ & $3.06 \pm 1.43$ & $4.27 \pm 2.16$ & $3.49 \pm 1.63$ & $5.12 \pm 2.56$ \\
\hline SUR (MIM) Time corr & $2.10 \pm 0.89$ & $2.77 \pm 0.83$ & $2.43 \pm 1.24$ & $3.30 \pm 1.43$ & $3.20 \pm 1.74$ & $4.66 \pm 1.81$ \\
\hline SUR (HERMES) Time corr & $2.06 \pm 0.81$ & $2.70 \pm 0.67$ & $2.63 \pm 1.40$ & $3.65 \pm 1.53$ & $3.20 \pm 1.74$ & $4.66 \pm 1.81$ \\
\hline SUR (MIM) + PVEC + Time corr & $2.60 \pm 1.17$ & $3.59 \pm 1.15$ & $2.80 \pm 1.35$ & $3.93 \pm 1.43$ & $3.47 \pm 1.79$ & $5.23 \pm 1.79$ \\
\hline SUR (HERMES) + PVEC + Time corr & $2.55 \pm 1.07$ & $3.42 \pm 0.85$ & $3.01 \pm 1.52$ & $4.23 \pm 1.46$ & $3.43 \pm 1.75$ & $5.06 \pm 1.72$ \\
\hline
\end{tabular}

KRAS gene amplification cases $(n=3)$ are grouped with wild type. Average metrics values after PVE and uptake time correction are provided for the derivative metrics tumor-to-liver ratio (SUVTLR) and tumor-to-blood ratio (SUR) as well 
Table 4 Statistics for the separation of KRAS missense mutations based on 2-[18F]FDG uptake when KRAS gene amplification is grouped with wild type for all 60 lesions in 39 interventional PET/CT scans

\begin{tabular}{|c|c|c|c|c|c|c|c|}
\hline \multirow[t]{2}{*}{ Parameter } & \multicolumn{2}{|c|}{ Mean } & \multicolumn{2}{|l|}{ Peak } & \multicolumn{2}{|l|}{ Max } & \multirow[t]{2}{*}{ Statistic } \\
\hline & MIM & HERMES & MIM & HERMES & MIM & HERMES & \\
\hline $\begin{array}{l}\text { SUV } \\
\text { No corr }\end{array}$ & 0.05 & 0.02 & 0.07 & 0.04 & 0.01 & $\begin{array}{l}0.01 \\
0.01 \\
0.692\end{array}$ & $\begin{array}{l}\text { Stud. } t \text { test } \\
\text { Wilc. R. Sum } \\
\text { AUC }\end{array}$ \\
\hline $\begin{array}{l}\text { SUV } \\
\text { PVE corr }\end{array}$ & 0.03 & 0.01 & 0.03 & 0.02 & 0.004 & $\begin{array}{l}0.006 \\
0.005 \\
0.715\end{array}$ & $\begin{array}{l}\text { Stud. } t \text { test } \\
\text { Wilc. R. Sum } \\
\text { AUC }\end{array}$ \\
\hline $\begin{array}{l}\text { SUVTLR } \\
\text { PVE corr }\end{array}$ & 0.02 & 0.02 & 0.02 & 0.02 & 0.006 & $\begin{array}{l}0.009 \\
0.004 \\
0.722\end{array}$ & $\begin{array}{l}\text { Stud. } t \text { test } \\
\text { Wilc. R. Sum } \\
\text { AUC }\end{array}$ \\
\hline $\begin{array}{l}\text { SUV } \\
\text { PVE + time corr }\end{array}$ & 0.03 & 0.01 & 0.03 & 0.02 & 0.005 & $\begin{array}{l}0.006 \\
0.005 \\
0.714\end{array}$ & $\begin{array}{l}\text { Stud. } t \text { test } \\
\text { Wilc. R. Sum } \\
\text { AUC }\end{array}$ \\
\hline $\begin{array}{l}\text { SUR } \\
\text { time corr }\end{array}$ & 0.004 & 0.002 & 0.02 & 0.01 & 0.003 & $\begin{array}{l}0.003 \\
0.002 \\
0.733\end{array}$ & $\begin{array}{l}\text { Stud. } t \text { test } \\
\text { Wilc. R. Sum } \\
\text { AUC }\end{array}$ \\
\hline $\begin{array}{l}\text { SUR } \\
\text { PVE + time corr }\end{array}$ & $\begin{array}{l}0.002 \\
0.003 \\
0.725\end{array}$ & $\begin{array}{l}0.0009 \\
0.0004 \\
0.770\end{array}$ & $\begin{array}{l}0.004 \\
0.004 \\
0.723\end{array}$ & $\begin{array}{l}0.003 \\
0.002 \\
0.738\end{array}$ & $\begin{array}{l}0.0005 \\
0.0005 \\
0.766\end{array}$ & $\begin{array}{l}0.0008 \\
0.001 \\
0.753\end{array}$ & $\begin{array}{l}\text { Stud. } t \text { test } \\
\text { Wilc. R. Sum } \\
\text { AUC }\end{array}$ \\
\hline
\end{tabular}

Student's $t$ test $p$ values are given for all metrics, while Wilcoxon rank sum test with continuity correction $p$ values (abbreviated to Wilc. R. Sum) and AUC are given as a second and third line number only for PVE- and time-corrected SUR (bottom row) and for the maximum values derived from the Hermes segmentation contours. Values obtained after PVE and uptake time correction as well as for derivative metrics as tumor-to-liver ratio (SUVTLR) and tumor-to-blood ratio (SUR) are provided

are shown in Fig. 4. The sensitivities and specificities for predicting KRAS mutations by the SUR-derived metrics are shown in Table 5.

Logistic regression curves for SUV $\mathrm{MAX}_{\text {with }}$ and without PVE and uptake time corrections and for $S_{U} R_{M A X}$ with both corrections are shown in Fig. 5. We see some dependence of the logistics regression optimal cutoff values, on the segmentation methods used. The optimal cutoff values for SUR after PVE and time correction for MIM versus Hermes segmentation are: 3.04 versus 3.11 for $\mathrm{SUR}_{\text {MEAN }}, 2.89$ versus 3.11 for $\mathrm{SUR}_{\text {PEAK }}$ and 4.09 ver-

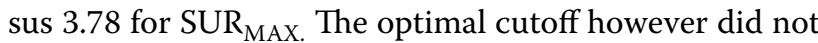
change between the two types of grouping for the KRAS gene amplification cases: with wild type as presented in
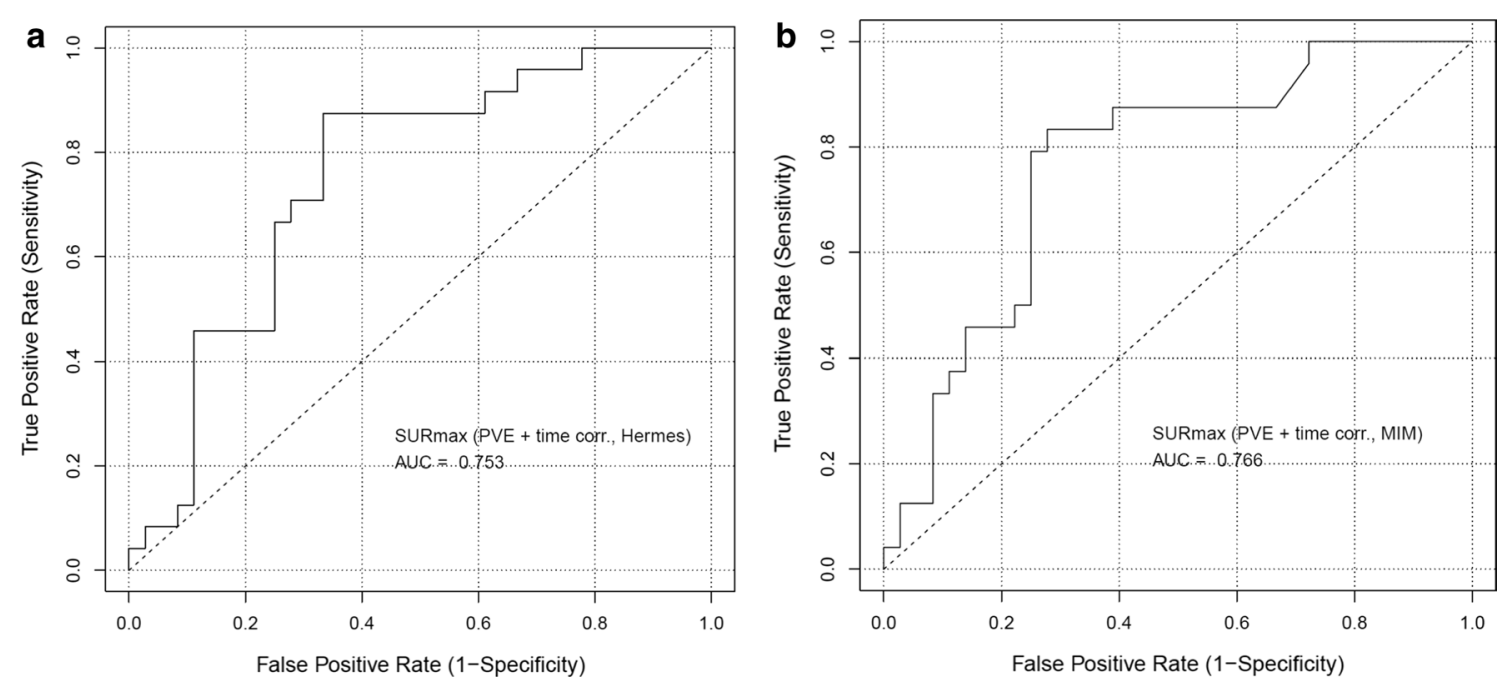

Fig. 4 Receiver operating characteristic (ROC) curves and AUC values for predicting CLM KRAS missense mutations (KRAS gene amplification is grouped with wild type) based on all 60 lesions and SUR $R_{\text {MAX }}$ with both corrections for Hermes (a, left) and MIM segmentations (b, right) 
Table 5 Specificities and sensitivities for predicting KRAS missense mutations based on all 60 lesions using SUR metrics after PVE and uptake time corrections

\begin{tabular}{|c|c|c|c|}
\hline & Specificity & Sensitivity & Sensitivity + Specificity \\
\hline \multicolumn{4}{|l|}{ SUR MEAN $_{\text {MEA }}$} \\
\hline MIM & 0.72 & 0.79 & 1.51 \\
\hline Hermes & 0.81 & 0.71 & 1.51 \\
\hline \multicolumn{4}{|l|}{ SUR PEAK } \\
\hline MIM & 0.67 & 0.83 & 1.50 \\
\hline Hermes & 0.67 & 0.88 & 1.54 \\
\hline \multicolumn{4}{|l|}{$S U R_{\text {MAX }}$} \\
\hline MIM & 0.72 & 0.83 & 1.56 \\
\hline Hermes & 0.67 & 0.88 & 1.54 \\
\hline
\end{tabular}

The rounded values obtained for contours drawn by different operators using different types of segmentation algorithms within different software platforms are listed

Fig. 5 and with KRAS+ as presented in Additional file 1: Fig. S2.

In 31/39 interventional PET/CT scans analyzed, the mutational status of the targeted lesion was determined from the specimen extracted from the same procedure. Applying the analysis for SUV $\mathrm{MAX}_{\text {ax }}$ only to the 31 lesions with accurately known KRAS mutational status also showed separation of the two groups when two remaining amplification cases are grouped with the wild type (average Student's $t$ test $p$ values between the two segmentations: 0.003 for SUV $\mathrm{MAX}_{\mathrm{M}}$ with no corrections, 0.020 after both corrections and 0.011 for SUR $\mathrm{MAX}_{\mathrm{X}}$ after both corrections).

If we remove the 12 lesions in the vicinity of previously treated parts of the liver and analyze the remaining 48 lesions, the Student's t test $p$ values for separating KRAS+ mutations from wild type and whole gene amplification based on maximum uptake are: 0.015 for SUV, 0.006 for $\mathrm{SUV}_{\mathrm{PVE}}, 0.008$ for SUVTLR, 0.005 for $\mathrm{SUV}_{\mathrm{PVE}+\text { time }}$

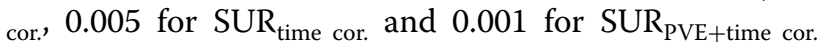
In this case, the mean AUCs between the two segmentations are 0.74 for $\mathrm{SUV}_{\mathrm{MAX}}$, PVE + Time corr and 0.77 for $\mathrm{SUR}_{\text {MAX,PVE+time cor }}$ If within this group of 48 lesions $K R A S$ whole gene amplification cases are grouped with $K R A S+$, statistical significance is lost.

When the three KRAS gene amplification cases are grouped with KRAS missense mutations, the $p$ values are higher and the specificity and sensitivity and the AUC values are lower (see Additional file 1: Tables S1, S2 and Fig. S1).

\section{Discussion}

For the investigated patient cohort, applying PVE corrections to the SUV leads to better separation of the KRAS mutations for all three SUV- and SUR-based metrics: mean, peak and max (Tables 3 and 4, Figs. 4 and 5). Applying uptake time correction leads to noticeably better separation of the two groups only for the SUR metrics but not for the SUV metrics (Table 4). PVE-corrected tumor-to-liver ratios, SUVTLR, performed similarly to PVE-corrected SUV. Using the uptake time-corrected tumor-to-blood ratio, SUR, both with and without PVEC result in better separation of the two groups than PVEand uptake time-corrected SUV. This indicates that uptake time variability is not the only relevant factor affecting the correlation between the chosen uptake metric and the tumor's metabolic rate. The removal of inaccuracies related to injected activity due to the residual activity assumption, scanner calibration and body mass/
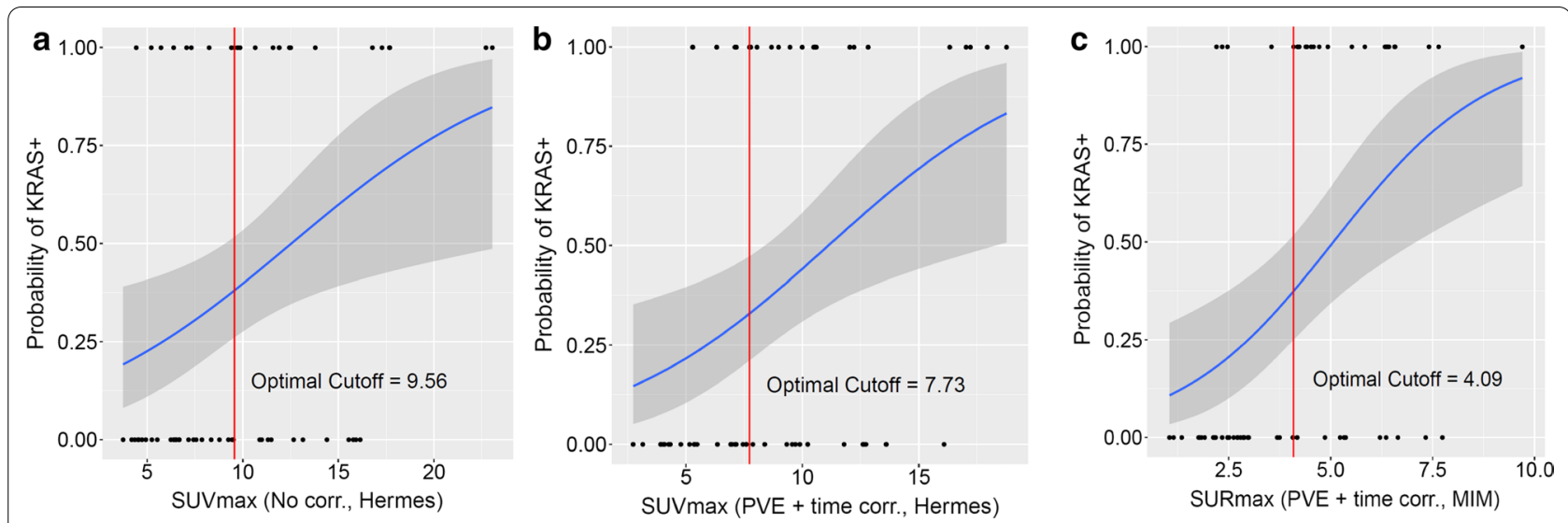

Fig. 5 Logistic regression curves based on all 60 lesions for SUV MAX without any corrections (a), with both PVE and uptake time corrections (b), and

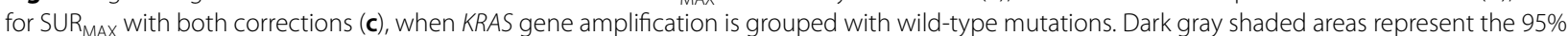
confidence intervals around the probability values. Individual data points are shown with dots at probability levels of 0.00 and 1.00 
weight as well as accounting for inter-subject variability of the arterial tracer supply as achieved by the use of the SUR metric is of key importance as well [40]. In fact, the logistic regression analysis (Fig. 5) shows that for the current patient cohort only the PVE- and uptake time-cor-

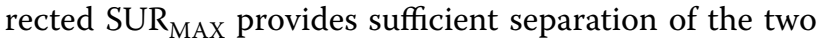
groups to make mutation predictions from the 2-[18F] FDG uptake in PET images.

Using SUR $\mathrm{MAX}_{\mathrm{MA}}$ and applying the corrections described above seem to have allowed for better separation of KRAS mutated from wild-type CRC liver lesions than that demonstrated previously [24-27, 30] (Table 4). When KRAS gene amplification is grouped with wild type, the PVE- and uptake time-corrected $S_{\text {UR }}$ MAX gives the highest predictive value: Between the two segmentations, the mean specificity is 0.694 and the mean sensitivity is 0.854 (sum 1.548, Table 5). For SUR MEAN $_{\text {and }}$ SUR $_{\text {PEAK }}$, the mean specificity + sensitivity is slightly lower: 1.51 and 1.52 .

Another factor we consider helpful in achieving better separation of the $K R A S$-mutated cases is an increased accuracy in labeling the mutational status. In 31/39 interventional PET/CT scans analyzed, the mutational status of the targeted lesion was determined from the specimen extracted from the same procedure. According to previous publications, about $6-10 \%$ of the remaining 29 lesions may have mutational status different from that determined in other lesions of the same patient [17]. Assuming that the overall discordance rate is approximately $10 \%$ [17], and since the mutational status of $31 / 60$ lesions is precisely known, only $5 \%(0.1 *(60-31)=2.9)$ of the 60 lesions may be potentially mislabeled. This decreases in half the general discordance rate. Thus, including target tumors with a precisely known mutational status (52\% in this cohort) increases the accuracy of the dataset and may have contributed to better separation of the two mutations.

In our dataset, the KRAS missense mutations seem better separated (lower $p$ values, higher AUC in Table 4 and Fig. 4) when the cases with KRAS gene amplification ( 3 of the 32 cases tested by MSK-IMPACT) are grouped with the wild type. This is understandable since KRASmutated cells were shown to exhibit enhanced glucose uptake [20] and these missense mutations were also found to be mutually exclusive with $K R A S$ whole gene amplification in this as well as in other patient cohorts [48]. The optimal cutoff values in the logistic regression analysis were not affected by reassigning the KRAS gene amplification cases to the KRAS mutant group (Fig. 5 and Additional file 1: Fig. S2) probably due to the small number of gene amplification cases. The mean specificity and sensitivity between the two segmentations for PVE- and time-corrected SUR $\mathrm{MAX}_{\text {in }}$ this case are 0.69 and 0.77 , respectively (Additional file 1: Table S2), compared to 0.69 and 0.85 for grouping with the wild type (Table 5).

One study has shown association of KRAS gene amplification with lack of response to anti-EGFR therapy in all four gene amplification cases of 53 non-responding cases; however, according to the authors this was not statistically significant and for these cases it was mutually exclusive with KRAS missense mutations [48]. KRAS gene amplification was seen in $0.7 \%$ of cases with "de novo resistance to anti-EGFR treatment" [49]. However, while KRAS amplification is a recurrent event in CRC and designated as likely oncogenic by the OncoKB knowledge base [50], at present, KRAS amplification per se is not a contraindication for anti-EGFR therapy [51] and its clinical significance remains uncertain.

In the investigated cohort, most of the observed KRAS missense mutations are in codon $12(n=8)$, but codons $13(n=4), 61(n=2)$ and $146(n=1)$ were also represented. While for mutations in codon 61 the reduction in response rate to cetuximab plus chemotherapy compared to wild type was found to be significant, that for mutations in codon 146 was not significant for a small number of cases [9]. Also, KRAS G13D mutations were shown to have potential positive effect on survival compared to other KRAS mutations in a retrospective study; however; further scrutiny of this effect is needed [52, 53]. Therefore, if the above findings of the effect of KRAS missense mutation location are confirmed, investigations of the effect of mutation location on 2-[18F]FDG uptake with larger patient cohorts harboring such mutations would be justified.

The ROC and logistic regression curves (Figs. 4 and 5) show that some of the 2-[18F]FDG uptake-based metrics presented have potential to predict KRAS missense mutations in CLM. From the investigated metrics, PVEand uptake time-corrected $\mathrm{SUR}_{\mathrm{MAX}}$ results in the highest AUC, specificity and sensitivity and therefore seems most promising for mutational status characterization. Making such a prediction based on previous or on intraprocedural PET images can prompt the interventional radiologist to biopsy lesions which may harbor mutations potentially different from those previously established or among different lesions. In this way, early predictions of CLM KRAS mutations, especially for cases with multiple lesions, would allow optimization of immediate interventions $[11,14]$ as well as of future treatments [6-10].

To further establish 2-[18F]FDG uptake as a biomarker of KRAS+ status in CLM, it is important to address the limitations of the presented investigation. Several steps in that direction can be taken. One would be to reduce the uncertainty in SUV by requiring residual activity measurement for each patient injection. Another would be including more lesions with specifically known 
mutational status to further reduce the labeling error and improve statistics. Use of SUR eliminates uncertainties related to injected activity and other technical and patient-related factors. While several studies have shown that using the SUR-based uptake time correction leads to finding better defined correlations by reducing variance in uptake, this work extends the applicability of this approach to larger uptake time differences.

\section{Conclusion}

Reducing the number of potentially mislabeled cases by focusing predominantly on lesions with genomic profile obtained from 2-[18F]FDG PET/CT-guided biopsies, applying partial volume effect and uptake time corrections, and using the ratio of maximum tumor to mean blood uptake in PET/CT scans, allowed for a highly statistically significant separation of colorectal adenocarcinoma liver lesions expressing KRAS missense mutations. Future testing with a larger patient cohort and implementation of these algorithms in the clinic may allow to assign probabilities for the KRAS mutation status of such lesions from the PET images. This may lead to further optimization of interventions and therapy for metastatic colorectal adenocarcinoma in the liver.

\section{Supplementary information}

Supplementary information accompanies this paper at https://doi. org/10.1186/s13550-020-00707-0.

Additional file 1: Results when the KRAS gene amplification cases are grouped with KRAS missense mutation cases.

\section{Abbreviations}

AUC: Area under the curve; BRAF: B-Raf; CRC: Colorectal cancer; CLM: Colorectal adenocarcinoma liver metastases; EGFR: Epidermal growth factor receptor; HER2: Human epidermal growth factor receptor-2; FOV: Field of view; 2- $\left[{ }^{18} \mathrm{~F}\right]$ FDG: 2-Deoxy-2-[18F]fluoro-D-glucose; KRAS: Kristen rat sarcoma viral gene; KRAS+ : KRAS missense mutation; NSCLC: Non-small cell lung cancer; PET: Positron emission tomography; PSF: Point-spread function; PVE: Partial volume effect; RC: Recovery coefficient; ROC: Receiver operating characteristic; SBR: Sphere-to-background activity ratio; SUV: Standard uptake values; SUR: Tumor-

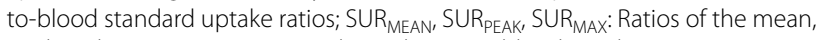
peak and maximum tumor uptake to the mean blood uptake; SUVTLR: Tumorto-liver uptake ratio; VOI: Volume of interest.
\end{abstract}

\section{Acknowledgements}

We gratefully acknowledge the diligent support from the radiology, molecular pathology, and anesthesia staff and technologists, as well as from the members of the Molecular Diagnostics Service in the Department of Pathology.

\section{Authors' contributions}

CTS and SBS had performed most of the PET/CT-guided interventions. ASK, $\mathrm{SD}, \mathrm{CTS}, \mathrm{SBS}, \mathrm{HS}, \mathrm{JLH}, \mathrm{RY}, \mathrm{EK}, \mathrm{PZ}$ and MEM participated in the design of the investigation. MP segmented the CLM using Hermes and performed most of the data analysis with guidance from ASK. OT segmented the CLM using MIM and performed a significant part of the data analysis and verification. JvdH advised on the implementation of the uptake time correction and derived the cold wall correction for the PVE recovery coefficients. HK helped with patent data extraction and verification based on patient records and with the data analysis. ZZ performed the Wilcoxon rank sum test, the ROC AUC analysis and the logistic regression statistical analysis. SD double-checked KRAS mutation labeling by independent and additional genomic record extraction. DL developed the code for automatic header information extraction (DBbrowser) and helped MP and OT to use it for this paper. PZ assisted in performing micro-CT of the NEMA phantom. JL performed the database searches to identify the patients for this study. EK helped with the lesion segmentation in MIM. CC helped with the patient data extraction and analysis. ASK organized the investigation, designed the PVE correction in close collaboration with JvdH, performed the recovery coefficient measurements, directed and participated in the data analysis and wrote the manuscript. All authors read and approved the final manuscript.

\section{Funding}

This work was funded in part by the Department of Medical Physics at MSKCC, by the Marie-Josée and Henry R. Kravis Center for Molecular Oncology, and by the National Cancer Institute Cancer Center Core Grant No. P30-CA008748.

\section{Availability of data and materials}

The datasets generated and/or analyzed during the current study are available from the corresponding author on reasonable request.

\section{Ethics approval and consent to participate}

The Institutional Review Board/Privacy Board-B of Memorial Sloan Kettering Cancer Center (IRB) approved the study and waived the need for informed consent. The IRB reviews and oversees human subject research to assure that it meets the ethical principles embodied in the Belmont Report and complies with US federal regulations that pertain to human subject protection at 45 CFR 46 and 21 CFR 50 and 56, AAHRPP standards, and other pertinent regulations, guidance and state and local laws.

\section{Consent for publication}

Not applicable. See above.

\section{Competing interests}

The authors declare that they have no competing interests. SBS is a consultant for Varian, Johnson \& Johnson, Aperture, XACT Robotics and Endoways, has research support from GE Healthcare, Elesta, Johnson \& Johnson and AngioDynamics and is a shareholder in Johnson \& Johnson and Aperture. MEM received speaker honoraria from Bristol Myers Squibb and Siemens and research support from Siemens, unrelated to the work considered.

\section{Author details}

${ }^{1}$ Memorial Sloan Kettering Cancer Center, 1275 York Avenue, New York, NY 10065, USA. ${ }^{2}$ Cornell University, Ithaca, NY 14850, USA. ${ }^{3}$ Vassar Brothers Medical Center, Poughkeepsie, NY 12601, USA. ${ }^{4}$ Lebedev Physical Institute RAS, Moscow, Russia 119991. ${ }^{5}$ Institute of Radiopharmaceutical Cancer Research, Helmholtz-Zentrum Dresden-Rossendorf, 01328 Dresden, Germany. ${ }^{6}$ Department of Radiology, Memorial Sloan Kettering Cancer Center, 1275 York Avenue, New York, NY 10065, USA. ${ }^{7}$ Department of Epidemiology \& Biostatistics, Memorial Sloan Kettering Cancer Center, 1275 York Avenue, New York, NY 10065, USA. ${ }^{8}$ Department of Pathology, Memorial Sloan Kettering Cancer Center, 1275 York Avenue, New York, NY 10065, USA. ${ }^{9}$ Technology Division, Memorial Sloan Kettering Cancer Center, 1275 York Avenue, New York, NY 10065, USA. ${ }^{10}$ Department of Medicine, Memorial Sloan Kettering Cancer Center, 1275 York Avenue, New York, NY 10065, USA.

Received: 30 June 2020 Accepted: 21 September 2020

Published online: 23 November 2020

\section{References}

1. Fleuren GJ, Gorter A, Kuppen PJ, Litvinov S, Warnaar SO. Tumor heterogeneity and immunotherapy of cancer. Immunol Rev. 1995;145:91-122. https://doi.org/10.1111/j.1600-065x.1995.tb00078.x.

2. Gerlinger M, Rowan AJ, Horswell S, Larkin J, Endesfelder D, Gronroos E, et al. Intratumor heterogeneity and branched evolution revealed by multiregion sequencing. N Engl J Med. 2012;366:883-92. https://doi. org/10.1056/NEJMoa1113205. 
3. Gillies RJ, Kinahan PE, Hricak H. Radiomics: images are more than pictures, they are data. Radiology. 2016;278:563-77. https://doi.org/10.1148/radio I.2015151169.

4. Yip SS, Aerts HJ. Applications and limitations of radiomics. Phys Med Biol. 2016;61:R150-66. https://doi.org/10.1088/0031-9155/61/13/R150.

5. Kishore SA, Drabkin MJ, Sofocleous CT. Fluorodeoxyglucose-PET for ablation treatment planning, intraprocedural monitoring, and response. J PET Clin. 2019;14:427-36. https://www.sciencedirect.com/science/article/pii/ S1556859819300483?via\%3Dihub.

6. Lievre A, Bachet JB, Le Corre D, Boige V, Landi B, Emile JF, et al. KRAS mutation status is predictive of response to cetuximab therapy in colorectal cancer. Cancer Res. 2006;66:3992-5. https://doi.org/10.1158/0008-5472. CAN-06-0191.

7. Lievre A, Bachet J-B, Boige V, Cayre A, Le Corre D, Buc E, et al. KRAS mutations as an independent prognostic factor in patients with advanced colorectal cancer treated with cetuximab. J Clin Oncol. 2008:26:374-9. https://doi.org/10.1200/jco.2007.12.5906.

8. Amado RG, Wolf M, Peeters M, Van Cutsem E, Siena S, Freeman DJ, et al. Wild-type KRAS is required for panitumumab efficacy in patients with metastatic colorectal cancer. J Clin Oncol. 2008;26:1626-34. https://doi. org/10.1200/JCO.2007.14.7116.

9. De Roock W, Claes B, Bernasconi D, De Schutter J, Biesmans B, Fountzilas $\mathrm{G}$, et al. Effects of KRAS, BRAF, NRAS, and PIK3CA mutations on the efficacy of cetuximab plus chemotherapy in chemotherapy-refractory metastatic colorectal cancer: a retrospective consortium analysis. Lancet Oncol. 2010;11:753-62. https://doi.org/10.1016/S1470-2045(10)70130-3.

10. Misale S, Yaeger R, Hobor S, Scala E, Janakiraman M, Liska D, et al. Emergence of KRAS mutations and acquired resistance to anti-EGFR therapy in colorectal cancer. Nature. 2012;486:532-6. https://doi.org/10.1038/natur e11156.

11. Shady W, Petre EN, Vakiani E, Ziv E, Gonen M, Brown KT, et al. Kras mutation is a marker of worse oncologic outcomes after percutaneous radiofrequency ablation of colorectal liver metastases. Oncotarget. 2017;8:66117-27. https://doi.org/10.18632/oncotarget.19806.

12. Vauthey J-N, Zimmitti G, Kopetz SE, Shindoh J, Chen SS, Andreou A, et al. RAS mutation status predicts survival and patterns of recurrence in patients undergoing hepatectomy for colorectal liver metastases. Ann Surg. 2013;258:619-27. https://doi.org/10.1097/sla.0b013e3182a5025a.

13. Arslan E, Aksoy T, Gürsu RU, Dursun N, Çakar E, Çermik TF. The prognostic value of 18F-FDG PET/CT and KRAS mutation in colorectal cancers. Mol Imaging Radionuclide Ther. 2020;29:17-24. https://doi.org/10.4274/mirt galenos.2019.33866.

14. Ziv E, Erinjeri JP, Yarmohammadi $H$, Boas FE, Petre EN, Gao S, et al. Lung adenocarcinoma: predictive value of KRAS mutation status in assessing local recurrence in patients undergoing image-guided ablation. Radiology. 2017;282:251-8. https://doi.org/10.1148/radiol.2016160003.

15. Brannon AR, Vakiani E, Sylvester BE, Scott SN, McDermott G, Shah RH, et al. Comparative sequencing analysis reveals high genomic concordance between matched primary and metastatic colorectal cancer lesions. Genome Biol. 2014;15:454. https://doi.org/10.1186/s13059-014-0454-7.

16. Han CB, Li F, Ma JT, Zou HW. Concordant KRAS mutations in primary and metastatic colorectal cancer tissue specimens: a meta-analysis and systematic review. Cancer Invest. 2012;30:741-7. https://doi. org/10.3109/07357907.2012.732159.

17. Mao C, Wu XY, Yang ZY, Threapleton DE, Yuan JQ, Yu YY, et al. Concordant analysis of KRAS, BRAF, PIK3CA mutations, and PTEN expression between primary colorectal cancer and matched metastases. Sci Rep. 2015;5:8065. https://doi.org/10.1038/srep08065.

18. Corcoran RB, André T, Atreya CE, Schellens JHM, Yoshino T, Bendell $\mathrm{JC}$, et al. Combined BRAF, EGFR, and MEK inhibition in patients with BRAFV600E-mutant colorectal cancer. Cancer Discov. 2018;8:428-43. https://doi.org/10.1158/2159-8290.cd-17-1226.

19. Russo M, Crisafulli G, Sogari A, Reilly NM, Arena S, Lamba S, et al. Adaptive mutability of colorectal cancers in response to targeted therapies. Science. 2019;366:1473-80. https://doi.org/10.1126/science.aav4474.

20. Yun J, Rago C, Cheong I, Pagliarini R, Angenendt P, Rajagopalan H, et al. Glucose deprivation contributes to the development of KRAS pathway mutations in tumor cells. Science. 2009;325:1555-9. https://doi. org/10.1126/science.1174229.

21. Caicedo C, Garcia-Velloso MJ, Lozano MD, Labiano T, Vigil Diaz C, LopezPicazo JM, et al. Role of $\left[{ }^{18} \mathrm{~F}\right] \mathrm{FDG}$ PET in prediction of KRAS and EGFR mutation status in patients with advanced non-small-cell lung cancer. Eur J Nucl Med Mol Imaging. 2014;41:2058-65. https://doi.org/10.1007/ s00259-014-2833-4.

22. Yip SS, Kim J, Coroller TP, Parmar C, Velazquez ER, Huynh E, et al. Associations between somatic mutations and metabolic imaging phenotypes in non-small cell lung cancer. J Nucl Med. 2017;58:569-76. https://doi. org/10.2967/jnumed.116.181826.

23. Krikelis D, Skoura E, Kotoula V, Rondogianni P, Pianou N, Samartzis A, et al. Lack of association between KRAS mutations and 18F-FDG PET/ CT in Caucasian metastatic colorectal cancer patients. Anticancer Res. 2014;34:2571-9. https://pubmed.ncbi.nlm.nih.gov/24778079/.

24. Chen SW, Chiang HC, Chen WT, Hsieh TC, Yen KY, Chiang SF, et al. Correlation between $\mathrm{PET} / \mathrm{CT}$ parameters and KRAS expression in colorectal cancer. Clin Nucl Med. 2014;39:685-9. https://doi.org/10.1097/RLU.00000 00000000481.

25. Chen SW, Lin CY, Ho CM, Chang YS, Yang SF, Kao CH, et al. Genetic alterations in colorectal cancer have different patterns on 18F-FDG PET/CT. Clin Nucl Med. 2015;40:621-6. https://doi.org/10.1097/RLU.000000000000083 0 .

26. Chen SW, Shen WC, Chen WT, Hsieh TC, Yen KY, Chang JG, et al. Metabolic imaging phenotype using radiomics of [(18)F]FDG PET/CT associated with genetic alterations of colorectal cancer. Mol Imaging Biol. 2018;21:183-90. https://doi.org/10.1007/s11307-018-1225-8.

27. Lovinfosse $P$, Koopmansch B, Lambert F, Jodogne S, Kustermans G, Hatt $M$, et al. (18)F-FDG PET/CT imaging in rectal cancer: relationship with the RAS mutational status. Br J Radiol. 2016;89:20160212. https://doi. org/10.1259/bjr.20160212.

28. Lee JH, Kang J, Baik SH, Lee KY, Lim BJ, Jeon TJ, et al. Relationship between 18F-fluorodeoxyglucose uptake and V-Ki-Ras2 kirsten rat sarcoma viral oncogene homolog mutation in colorectal cancer patients: variability depending on C-reactive protein level. Medicine (Baltimore). 2016;95:e2236. https://doi.org/10.1097/MD.0000000000002236.

29. Miles KA, Ganeshan B, Rodriguez-Justo M, Goh VJ, Ziauddin Z, Engledow A, et al. Multifunctional imaging signature for V-KI-RAS2 Kirsten rat sarcoma viral oncogene homolog (KRAS) mutations in colorectal cancer. J Nucl Med. 2014;55:386-91. https://doi.org/10.2967/jnumed.113.120485.

30. Kawada K, Toda K, Nakamoto Y, Iwamoto M, Hatano E, Chen F, et al. Relationship between 18F-FDG PET/CT Scans and KRAS mutations in metastatic colorectal cancer. J Nucl Med. 2015;56:1322-7. https://doi. org/10.2967/jnumed.115.160614.

31. Tatli S, Gerbaudo VH, Feeley CM, Shyn PB, Tuncali K, Silverman SG. PET/ CT-guided percutaneous biopsy of abdominal masses: initial experience. J Vasc Interv Radiol. 2011;22:507-14. https://doi.org/10.1016/j. jvir.2010.12.035

32. Shyn PB. Interventional positron emission tomography/computed tomography: state-of-the-art. Tech Vasc Interv Radiol. 2013;16:182-90. https://doi.org/10.1053/j.tvir.2013.02.014.

33. Solomon SB, Cornelis F. Interventional molecular imaging. J Nucl Med. 2016;57:493-6. https://doi.org/10.2967/jnumed.115.161190.

34. Cazzato RL, Garnon J, Ramamurthy N, Tsoumakidou G, Imperiale A, Namer IJ, et al. 18F-FDOPA PET/CT-guided radiofrequency ablation of liver metastases from neuroendocrine tumours: technical note on a preliminary experience. Cardiovasc Intervent Radiol. 2016;39:1315-21. https ://doi.org/10.1007/s00270-016-1334-1.

35. Fei B, Schuster DM. PET Molecular imaging-directed biopsy: a review. AJR Am J Roentgenol. 2017;209:255-69. https://doi.org/10.2214/AJR.17.18047

36. Fanchon LM, Apte A, Schmidtlein CR, Yorke E, Hu YC, Dogan S, et al. Evaluation of the tumor registration error in biopsy procedures performed under real-time PET/CT guidance. Med Phys. 2017;44:5089-95. https:// doi.org/10.1002/mp.12334.

37. Arcila M, Lau C, Nafa K, Ladanyi M. Detection of KRAS and BRAF mutations in colorectal carcinoma: Roles for high-sensitivity locked nucleic acid-PCR sequencing and broad-spectrum mass spectrometry genotyping. J Mol Diagn. 2011;13:64-73. https://doi.org/10.1016/j.jmoldx.2010.11.005.

38. Cheng DT, Mitchell TN, Zehir A, Shah RH, Benayed R, Syed A, et al. Memorial Sloan Kettering-integrated mutation profiling of actionable cancer targets (MSK-IMPACT): a hybridization capture-based next-generation sequencing clinical assay for solid tumor molecular oncology. J Mol Diagn. 2015;17:251-64. https://doi.org/10.1016/j.jmoldx.2014.12.006. 
39. van den Hoff J, Lougovski A, Schramm G, Maus J, Oehme L, Petr $J$, et al. Correction of scan time dependence of standard uptake values in oncological PET. EJNMMI research. 2014;4:18. https://doi. org/10.1186/2191-219X-4-18.

40. van den Hoff J, Oehme L, Schramm G, Maus J, Lougovski A, Petr J, et al. The PET-derived tumor-to-blood standard uptake ratio (SUR) is superior to tumor SUV as a surrogate parameter of the metabolic rate of FDG. EJNMMI Res. 2013;3:77. https://doi.org/10.1186/2191-219X-3-77.

41. NEMA NU 2-2018, Performance Measurements of Positron Emission Tomographs (PET). Rosslyn, VA: National Electrical Manufacturers Association; 2018. https://www.nema.org/standards/view/Performance-Measu rements-of-Positron-Emission-Tomographs.

42. Hofheinz F, Dittrich S, Pötzsch C, van den Hoff J. Effects of cold sphere walls in PET phantom measurements on the volume reproducing threshold. Phys Med Biol. 2010;55:1099-113. https://doi. org/10.1088/0031-9155/55/4/013.

43. Hofheinz F, Butof R, Apostolova I, Zophel K, Steffen IG, Amthauer H, et al. An investigation of the relation between tumor-to-liver ratio (TLR) and tumor-to-blood standard uptake ratio (SUR) in oncological FDG PET. EJNMMI Res. 2016;6:19. https://doi.org/10.1186/s13550-016-0174-y.

44. Hofheinz F, Hoffvd J, Steffen IG, Lougovski A, Ego K, Amthauer H, et al. Comparative evaluation of SUV, tumor-to-blood standard uptake ratio (SUR), and dual time point measurements for assessment of the metabolic uptake rate in FDG PET. EJNMMI Res. 2016;6:53. https://doi. org/10.1186/s13550-016-0208-5.

45. Hofheinz F, Apostolova I, Oehme L, Kotzerke J, van den Hoff J. Test-retest variability in lesion SUV and lesion SUR in (18)F-FDG PET: an analysis of data from two prospective multicenter trials. J Nucl Med. 2017;58:1770-5. https://doi.org/10.2967/jnumed.117.190736.

46. Butof R, Hofheinz F, Zophel K, Schmollack J, Jentsch C, Zschaeck S, et al. Prognostic Value of standardized uptake ratio in patients with trimodality treatment of locally advanced esophageal carcinoma. J Nucl Med. 2019;60:192-8. https://doi.org/10.2967/jnumed.117.207670.
47. Hofheinz F, Li Y, Steffen IG, Lin Q, Lili C, Hua W, et al. Confirmation of the prognostic value of pretherapeutic tumor SUR and MTV in patients with esophageal squamous cell carcinoma. Eur J Nucl Med Mol Imaging. 2019;46:1485-94. https://doi.org/10.1007/s00259-019-04307-6.

48. Valtorta E, Misale S, Sartore-Bianchi A, Nagtegaal ID, Paraf F, Lauricella $C$, et al. KRAS gene amplification in colorectal cancer and impact on response to EGFR-targeted therapy. Int J Cancer. 2013;133:1259-65. https ://doi.org/10.1002/ijc.28106.

49. Misale S, Di Nicolantonio F, Sartore-Bianchi A, Siena S, Bardelli A. Resistance to anti-EGFR therapy in colorectal cancer: from heterogeneity to convergent evolution. Cancer Discov. 2014;4:1269-80. https://doi. org/10.1158/2159-8290.CD-14-0462.

50. Chakravarty D, Gao J, Phillips S, Kundra R, Zhang H, Wang J, et al. OncoKB: a precision oncology knowledge base. JCO precision oncology. 2017;1:1-16. https://doi.org/10.1200/po.17.00011.

51. Benson AB, Venook AP, Al-Hawary MM, Cederquist L, Chen Y-J, Ciombor KK, et al. NCCN guidelines insights: colon cancer, version 2.2018. J Natl Compr Cancer Netw. 2018;16:359-69. https://doi.org/10.6004/jnccn .2018.0021.

52. De Roock W, Jonker DJ, Di Nicolantonio F, Sartore-Bianchi A, Tu D, Siena S, et al. Association of KRAS p.G13D mutation with outcome in patients with chemotherapy-refractory metastatic colorectal cancer treated with cetuximab. JAMA. 2010;304:1812-20. https://doi.org/10.1001/ jama.2010.1535.

53. De Roock W, De Vriendt V, Normanno N, Ciardiello F, Tejpar S. KRAS, BRAF, PIK3CA, and PTEN mutations: implications for targeted therapies in metastatic colorectal cancer. Lancet Oncol. 2011;12:594-603. https://doi. org/10.1016/S1470-2045(10)70209-6.

\section{Publisher's Note}

Springer Nature remains neutral with regard to jurisdictional claims in published maps and institutional affiliations.

\section{Submit your manuscript to a SpringerOpen ${ }^{\odot}$ journal and benefit from:}

- Convenient online submission

- Rigorous peer review

- Open access: articles freely available online

- High visibility within the field

- Retaining the copyright to your article

Submit your next manuscript at $\boldsymbol{\nabla}$ springeropen.com 